\title{
SYNTHESIS OF SOME NEW DERIVATIVES OF ISOINDOLINE-1,3-DIONE NUCLEUS FOR ANTIHYPERGLYCEMIC EVALUATION Ibrahim Hassan Eissa
}

Pharmaceutical Chemistry Department, Faculty of Pharmacy, Al-Azhar University, Cairo, Egypt

\begin{abstract}
:
In order to produce potent new leads for antidiabetic drugs, a new series of isoindoline-1,3-dione analogues bearing aryl sulfonylurea moieties were synthesized and screened for their antihyperglycemic activity. Some of newly synthesized compounds were identified as active antihyperglycemic agents. Compounds $N$-(cyclohexylcarbamoyl)-4-[2(1,3-dioxoisoindolin-2-yl) ethyl]benzenesulfon-amide(VII $)_{\mathbf{0}}, N$-(cyclopentylcabamoyl)-4-[2(1,3-dioxoiso- indolin-2-yl)ethyl] benzene sulfonamide (VII $), N$-(butylcabamoyl)-4-[2-(1,3dioxoisoindolin-2-yl)ethyl]benzenesulfonamide(VII $\left.\mathbf{q}_{\mathbf{q}}\right), N$-(cyclohexylcarbamoyl) -4- [ ( $1,3-$ dioxo - isoindolin- 2 - yl ) methyl ] benzenesulfonamide $\left(\mathbf{V I I}_{\mathbf{h}}\right)$ and $\mathrm{N}$-(propylcabamoyl)-4[2-(1,3-dioxoisoindolin-2-yl)ethyl]benzenesulfonamide $\left(\mathbf{V I I}_{\mathbf{r}}\right)$ proved to be the most active members of this study, as compered to to the reference gliclazide. They showed serum glucose reduction values of $52 \%, 48 \% 45 \%, 44 \%$ and $44 \%$, respectively. The detailed synthesis and biological screening data are reported.
\end{abstract}

Keywords: Isoindoline-1,3-diones ; Sulfonylurea; Antihyperglycemic activity.

\section{INTRODUCTION}

It is clear that isoindoline-1,3-dione nucleus is the backbone of many bioactive compounds that show versatile biological and pharmacological activities (Chan, et al., 2009; Miyachi, et al., 1997; Zhao, et al., 2009; Yang, et al., 2010; Santos, et al., 2009). Potential activity of isoindoline-1,3-dione as antihyperglycemic is well known as compounds I, II, III and IV (Mahapatra, et al., 2010; Abdel-Aziz, et al., 2011; Hashimoto,1998; Pascale, et al., 2010; Kim, et al., 2002).<smiles>O=C1c2ccccc2C(=O)N1CCc1ccccc1</smiles><smiles>O=C1c2ccccc2C(=O)N1Oc1cccc([N+](=O)[O-])c1</smiles><smiles>O=C1c2c(Cl)c(Cl)c(Cl)c(Cl)c2C(=O)N1CCCCc1ccccc1</smiles><smiles>CC(C)c1ccc(N2C(=O)c3ccccc3C2=O)cc1</smiles>

WHO estimates that more than 180 million people worldwide have diabetes. The incidence of diabetes is increasing with alarming mortality and morbidity and This number is likely to more than double (http://www. afro.who.int/en/clusters-a-programmes/dpc/noncomm unicable-diseases-managementndm/programme-components/ diabet- esmellitus.html. 2013). 
There is an increasing demand of new antidiabetic products due to the drawbacks associated with insulin and oral hypoglycemic agents actually available (Fertig, et al.,1995). In response to the enormity of the growing problem, efforts to identify and develop new pharmacological agents for type II diabetes have increased dramatically in recent years (http://diabetes manager.pbworks.com/w/page/ 17680289/Oral\% 20Pharmacological\%20 Agents\% 20for\% 20Type\% 202\% 20Diabetes, 2013). These efforts have resulted in the successful introduction of several new treatment options.

Currently, there is a very important class of oral pharmacological agents available to treat type II diabetes, sulfonylureas (Nadendla, 2005). More recently, $2^{\text {nd }}$ generation sulfonylureas are now widely used as an effective antihyperglycemic agents with a lower risk of adverse as compound V (Korytkowski, 2004; Kar, 2006).<smiles>CCC1=C(C)CN(C(=O)NCCc2ccc(S(=O)(=O)NC(=O)NC3CCC(C)CC3)cc2)C1=O</smiles>

From the previously mentioned findings, it was decided to synthesize some new compounds bearing both isoindoline-1,3-dione and sulfonylurea moieties hoping to obtain more effective antihyperglycemic agents.

For preparation of the newly synthesized compounds, the scheme 1 was adopted:<smiles>[R]c1c([R])c([R])c2c(c1[R])C(=O)OC2=O</smiles><smiles>N#Cc1ccc(S(N)(=O)=O)cc1</smiles><smiles>[R]c1c([R])c([R])c2c(c1[R])C(=O)N(c1ccc(S(N)(=O)=O)cc1)C2=O</smiles><smiles>[R]NC(=O)NS(=O)(=O)c1ccc(CN2C(=O)c3c([R])c([R])c([R])c([R])c3C2=O)cc1</smiles>
scheme 1: Synthesis of target compounds $\mathrm{VII}_{\mathrm{a}-\mathrm{u}}$

\section{Chemistry}

Reaction of sulfonamide derivatives with phthalic anhydride derivatives in glacial acetic acid gave compounds $\mathbf{V} \mathbf{I}_{\mathbf{a}-\mathbf{f}}$ that act as key compounds. The target compounds $\mathbf{V I I} \mathbf{I}_{\mathbf{a}-\mathbf{u}}$ 
were obtained through the reaction of compounds $\mathbf{V I}_{\mathbf{a}-\mathbf{f}}$ with alkyl/alicyclic isocyanate derivatives in dry acetone and anhydrous potassium carbonate.

\subsection{Experimental}

All melting points were taken on electrothermal (IA 9000 SERIS) digital melting point apparatus and are uncorrected. IR spectra were recorded on Pye Unicam SP 1000 IR spectrophotometer at Pharmaceutical analytical Unit, Faculty of Pharmacy, AlAzhar University. The ${ }^{1} \mathrm{H}$ NMR spectra were recorded in DMSO- $\mathrm{d}_{6}$ at $300 \mathrm{MHz}$ on a Varian Mercury VXR-300 NMR spectrometer at Research Services Unit, Faculty of Science, Cairo University. Chemical shifts were related to those of the solvent. Tetramethylsilane (TMS) was used as a standard. Mass spectra were recorded on Hewlett Packard 5988 spectrometer at Regional Center for Mycology and Biotechnology, Al-Azhar University. Microanalyses were carried out at Regional Center for Mycology and Biotechnology, Faculty of Science, AlAzhar University. Progresses of the reaction were monitored by TLC using TLC sheets precoated with UV fluorescent silica gel Merck 60 F254 plates and were visualized using UV lamp and dichloromethane : methanol 95:5 as mobile phase.

2.1.1. General procedure for the reaction of substituted isoindoline-1,3-dione with sulfonamide derivatives $\mathrm{VI}_{a-f}$ :

A mixture of sulfonamide derivative $(0.02 \mathrm{~mol})$ and phthalic anhydride $(0.02 \mathrm{~mol})$ was refluxed in glacial acetic acid $(50 \mathrm{~mL})$ for $10 \mathrm{~h}$. The solid obtained was filtered and washed with diluted ethanol to afford $\mathbf{V} \mathbf{I}_{\mathbf{a}-\mathbf{f}}$.

2.1.2. 4-(1,3-dioxoisoindolin-2-yl)benzenesulfonamide $\left(V I_{a}\right)$ : this compound has yield: $5.10 \mathrm{~g}(84 \%)$ and m.p: $340-341^{\circ} \mathrm{C}$ as reported (Ibrahim, 2010)

2.1.3. 4-(4,5,6,7-tetrabromo-1,3-dioxoisoindolin-2-yl)benzenesulfonamide $\quad\left(\boldsymbol{V I}_{\boldsymbol{b}}\right)$ : $\quad$ this compound has yield: $10.50 \mathrm{~g}(85 \%)$ and m.p: $345-346^{\circ} \mathrm{C}$ as reported (Ibrahim, 2010)

2.1.4. 4-[(1,3-dioxoisoindolin-2-yl)methyl]benzenesulfonamide $\left(V_{c}\right)$ :

$5.20 \mathrm{~g}$, (81\%); mp: $230-231^{\circ} \mathrm{C}$; IR: $\mathrm{NH}_{2} 3356,3257,2 \mathrm{CO} 1705 \mathrm{~cm}^{-1} ;{ }^{1} \mathrm{H}$ NMR (DMSO-d 6 ): $\delta 4.84$ (s, 2H, $\left.\mathrm{CH}_{2}\right), 7.30$ (s, 2H, NH) ( $\mathrm{D}_{2} \mathrm{O}$ exchangeable), 7.48-7.92(m, 8H, aromatic); Ms: $\mathrm{m} / \mathrm{z} 316\left(\mathrm{M}^{+}\right)(5.76 \%), 237\left(\mathrm{M}^{+}-\mathrm{SO}_{2} \mathrm{NH}_{2}\right)(10.96 \%), 161\left(\mathrm{M}^{+}-\mathrm{C}_{6} \mathrm{H}_{5} \mathrm{SO}_{2} \mathrm{NH}_{2}\right)$ (100\%). Anal. Calcd. for $\mathrm{C}_{15} \mathrm{H}_{12} \mathrm{~N}_{2} \mathrm{O}_{4} \mathrm{~S}$ : C, 56.95; H, 3.82; N, 8.86. Found: C, 56.55; H, 3.51; N, 8.45.

2.1.5. 4-[(4,5,6,7-tetrabromo-1,3-dioxoisoindolin-2-yl)methyl]benzenesulfona mide $\left(V I_{d}\right)$ : $10.40 \mathrm{~g}$, (83\%); mp: 338-339 ${ }^{\circ} \mathrm{C}$; IR: $\mathrm{NH}_{2} 3351,3249,2 \mathrm{CO} 1707 \mathrm{~cm}^{-1}$; $\quad{ }^{1} \mathrm{H}$ NMR (DMSO-d $\left.\mathrm{d}_{6}\right): \delta 4.82\left(\mathrm{~s}, 2 \mathrm{H}, \mathrm{CH}_{2}\right), 7.27(\mathrm{~s}, 2 \mathrm{H}, \mathrm{NH})\left(\mathrm{D}_{2} \mathrm{O}\right.$ exchangeable), 7.43-7.87(m, 4H, aromatic); Ms: $\mathrm{m} / \mathrm{z} 632 \quad\left(\mathrm{M}^{+}+5\right) \quad(3.54 \%), 549 \quad\left(\mathrm{M}^{+}-\mathrm{SO}_{2} \mathrm{NH}_{2}\right) \quad(20.76 \%), 160 \quad\left(\mathrm{M}^{+}-\right.$ $4 \mathrm{BrC}_{6} \mathrm{H}_{5} \mathrm{SO}_{2} \mathrm{NH}_{2}$ ) (100\%). Anal. Calcd. for $\mathrm{C}_{15} \mathrm{H}_{8} \mathrm{Br}_{4} \mathrm{~N}_{2} \mathrm{O}_{4} \mathrm{~S}: \mathrm{C}, 28.51 ; \mathrm{H}, 1.28 ; \mathrm{N}, 4.43$. Found: C, 28.88; H, 1.67; N, 4.11.

\subsubsection{4-[2-(1,3-dioxoisoindolin-2-yl)ethyl]benzenesulfonamide (VI $)_{e}$ :}

$5.41 \mathrm{~g},(82 \%)$; mp: $224-225^{\circ} \mathrm{C}$; IR: $\mathrm{NH}_{2} 3361,3271,2 \mathrm{CO} 1710 \mathrm{~cm}^{-1}$;

${ }^{1} \mathrm{H}$ NMR (DMSO-d ${ }_{6}$ ): $\delta 2.96$ (t, 2H, $\mathrm{CH}_{2}-\mathrm{ph}$ ), 3.80 (t, 2H, $\mathrm{CH}_{2}$-phthalimide), 7.25 (s, 2H, NH) $\left(\mathrm{D}_{2} \mathrm{O}\right.$ exchangeable), 7.38-7.88(m, 8H, aromatic); Ms: m/z $330\left(\mathrm{M}^{+}\right)(4.89 \%), 250\left(\mathrm{M}^{+}-\mathrm{SO}_{2} \mathrm{NH}_{2}\right)$ (23.49\%), $160\left(\mathrm{M}^{+}-\mathrm{CH}_{2} \mathrm{C}_{6} \mathrm{H}_{5} \mathrm{SO}_{2} \mathrm{NH}_{2}\right)(100 \%)$. Anal. Calcd. for $\mathrm{C}_{16} \mathrm{H}_{14} \mathrm{~N}_{2} \mathrm{O}_{4} \mathrm{~S}: \mathrm{C}, 58.17 ; \mathrm{H}$, 4.27 ; N, 8.48. Found: C, 58.45; H, 3.88; N, 8.05. 


\subsubsection{4-[2-(4,5,6,7-tetrabromo-1,3-dioxoisoindolin-2-yl)ethyl]benzenesulfon-amide (VI $)$ :} $10.78 \mathrm{~g},(84 \%)$; mp: 280-281 ${ }^{\circ} \mathrm{C}$; IR: $\mathrm{NH}_{2}$ 3346, 3253, 2CO $1707 \mathrm{~cm}^{-1}$; $\quad{ }^{1} \mathrm{H}$ NMR (DMSO-d ${ }_{6}$ ): $\delta 2.98\left(\mathrm{t}, 2 \mathrm{H}, \mathrm{CH}_{2}-\mathrm{ph}\right), 3.82\left(\mathrm{t}, 2 \mathrm{H}, \mathrm{CH}_{2}\right.$-phthalimide), $7.28(\mathrm{~s}, 2 \mathrm{H}, \mathrm{NH})\left(\mathrm{D}_{2} \mathrm{O}\right.$ exchangeable), 7.43-7.75(m, $4 \mathrm{H}$, aromatic). Anal. Calcd. for $\mathrm{C}_{16} \mathrm{H}_{10} \mathrm{Br}_{4} \mathrm{~N}_{2} \mathrm{O}_{4} \mathrm{~S}: \mathrm{C}, 29.75 ; \mathrm{H}$, 1.56; N, 4.34. Found: C, 29.43; H, 1.91; N, 4.75.

\subsubsection{General procedure for synthesis of $N$-substituted bnzensulfonamide derivatives VII ${ }_{a-}$} u:

Reaction mixture consisting of (0.002mol.) of $\mathbf{V I}_{\mathbf{a}-\mathbf{f}}$ and $(0.004 \mathrm{~mol} .0 .55 \mathrm{~g})$ of anhydrous potassium carbonate in $150 \mathrm{ml}$ of anhydrous acetone was stirred at refluxing temperature for about $1.5 \mathrm{~h}$. $(0.0025 \mathrm{~mol}$.) of the appropriate isocyanate was added dropwise to the reaction mixture. Refluxing and stirring were continued during the course of the addition and for an additional 16 hours. The acetone was removed by evaporation under reduced pressure, and about $750 \mathrm{ml}$ of water were added to dissolve the resulting residue. The solution was filtered. Acidification of the filtrate with $6 \mathrm{~N}$ aqueous hydrochloric acid caused the precipitation of the product which was collected by filtration. Crystallization of the filter cake from $90 \%$ aqueous ethanol yielded purified sulfonylurea derivatives.

2.1.9. $N$ - (cyclohexylcarbamoyl) - 4- (1,3-dioxoisoindolin- 2 -yl) benzenesulfon- amide $\left(\mathrm{VII}_{a}\right)$ :

$0.52 \mathrm{~g},(61 \%)$; mp: $291-292^{\circ} \mathrm{C}$; IR: $2 \mathrm{NH} 3328, \mathrm{CH}$-aliphatic 2928, $3 \mathrm{CO} 1624 \mathrm{~cm}^{-1} ;{ }^{1} \mathrm{H}$ NMR (DMSO-d $\left.\mathrm{d}_{6}\right): \delta 1.15-1.66(\mathrm{~m}, 10 \mathrm{H}$, cyclohexyl group), $3.22(\mathrm{~m}, 1 \mathrm{H}$, of cyclohexyl group), 5.51(s, 1H, NH-cyclohexyl) ( $\mathrm{D}_{2} \mathrm{O}$ exchangeable $), 6.30\left(\mathrm{~s}, 1 \mathrm{H}, \mathrm{NH}-\mathrm{SO}_{2}\right)\left(\mathrm{D}_{2} \mathrm{O}\right.$ exchangeable), 6.88-7.85(m, 8H, aromatic); Ms: m/z $427\left(\mathrm{M}^{+}\right)$(2.19\%), $302\left(\mathrm{M}^{+}-\mathrm{CONH}-\right.$ cyclohexyl) (100\%).Anal. Calcd. for $\mathrm{C}_{21} \mathrm{H}_{21} \mathrm{~N}_{3} \mathrm{O}_{5} \mathrm{~S}$ : C, 59.00; H, 4.95; N, 9.83. Found: C, 59.33; H, 4.51; N, 9.45 .

2.1.10. $N$-(cyclopentylcabamoyl)-4-(1,3-dioxoisoindolin-2-yl)benzenesulfona- mide (VII $\left.{ }_{b}\right)$ : 0.49 g, (59\%); mp: 283-284 ${ }^{\circ} \mathrm{C}$; IR: 2NH 3330, CH-aliphatic 2927, 3CO $1619 \mathrm{~cm}^{-1} ; \mathrm{Ms}: \mathrm{m} / \mathrm{z}$ $413\left(\mathrm{M}^{+}\right)(2.50 \%), 302\left(\mathrm{M}^{+}-\mathrm{CONH}-\right.$ cyclopentyl) (100\%). Anal. Calcd. for $\mathrm{C}_{20} \mathrm{H}_{19} \mathrm{~N}_{3} \mathrm{O}_{5} \mathrm{~S}$ : C, 58.10; H, 4.63; N, 10.16; Found: C, 58.44; H, 4.91; N, 4.25.

2.1.11. N-(Butylcabamoyl)-4-(1,3-dioxoisoindolin-2-yl)benzenesulfonamide (VII $)$ : $0.45 \mathrm{~g}$, (56\%); mp: 286-287 ${ }^{\circ} \mathrm{C}$;IR: 2NH 3330, CH-aliphatic 2929, 3CO $1625 \mathrm{~cm}^{-1}$; ${ }^{1} \mathrm{H}$ NMR (DMSO-d $\left.\mathrm{d}_{6}\right): \delta 0.85\left(\mathrm{t}, 3 \mathrm{H}, \mathrm{CH}_{3}\right), 1.45\left(\mathrm{~m}, 2 \mathrm{H}, \mathrm{C}_{2} \mathrm{CH}_{3}\right), 2.65\left(\mathrm{~m}, 2 \mathrm{H}, \mathrm{C}_{2} \mathrm{CH}_{2} \mathrm{CH}_{3}\right), 3.15(\mathrm{t}$, $2 \mathrm{H}, \mathrm{CH}_{2} \mathrm{CH}_{2} \mathrm{CH}_{2} \mathrm{CH}_{3}$ ), 5.52(s, $1 \mathrm{H}, \mathrm{NH}$-butyl) ( $\mathrm{D}_{2} \mathrm{O}$ exchangeable), 6.58(s, $1 \mathrm{H}, \mathrm{NH}-\mathrm{SO}_{2}$ ) ( $\mathrm{D}_{2} \mathrm{O}$ exchangeable), 7.43-7.88(m, 8H, aromatic); Ms: m/z $401\left(\mathrm{M}^{+}\right)(92.34 \%), 343\left(\mathrm{M}^{+}-\mathrm{H}-\right.$ butyl) (14.88\%), 303( $\mathrm{M}^{+}$- CONH-butyl) (29.32\%), 76(100\%), Anal. Calcd. for $\mathrm{C}_{19} \mathrm{H}_{19} \mathrm{~N}_{3} \mathrm{O}_{5} \mathrm{~S}$ : C, 56.85; H, 4.77; N, 10.47; Found: C, 56.44; H, 4.36; N, 9.99.

\subsubsection{2. $N$-(Propylcabamoyl)-4-(1,3-dioxoisoindolin-2-yl)benzenesulfonamide $\left(V_{1} I_{d}\right)$ :}

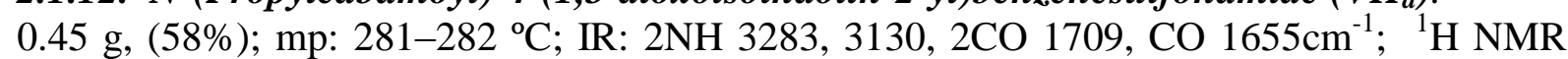
(DMSO-d $\mathrm{d}_{6}$ ): $\delta 0.90\left(\mathrm{t}, 3 \mathrm{H}, \mathrm{CH}_{3}\right), 1.49\left(\mathrm{~m}, 2 \mathrm{H}, \mathrm{C}_{2} \mathrm{CH}_{3}\right), 3.15\left(\mathrm{t}, 2 \mathrm{H}, \mathrm{C}_{2} \mathrm{CH}_{2} \mathrm{CH}_{3}\right), 5.51(\mathrm{~s}, 1 \mathrm{H}$, NH-propyl) ( $\mathrm{D}_{2} \mathrm{O}$ exchangeable), 6.81(s, 1H, NH-SO ${ }_{2}$ ) ( $\mathrm{D}_{2} \mathrm{O}$ exchangeable), 7.23-7.91(m, $8 \mathrm{H}$, aromatic); Anal. Calcd. for $\mathrm{C}_{18} \mathrm{H}_{17} \mathrm{~N}_{3} \mathrm{O}_{5} \mathrm{~S}$ : C, 55.80; H, 4.42; N, 10.85; Found: C, 55.65; $\mathrm{H}, 4.06 ; \mathrm{N}, 10.64$. 
2.1.13. $\quad N$-(cyclohexylcarbamoyl)-4-(4,5,6,7-tetrabromo-1,3-dioxoisoindolin-2yl)benzenesulfonamide (VII $)$ :

0.88 g, (60\%); mp: $275-276{ }^{\circ} \mathrm{C}$; IR: 2NH 3322, CH-aliphatic 2927, 3CO $1644 \mathrm{~cm}^{-1}$; ${ }^{1} \mathrm{H}$ NMR (DMSO-d $\left.\mathrm{d}_{6}\right): \delta 1.31-1.65(\mathrm{~m}, 10 \mathrm{H}$, cyclohexyl group), $3.33(\mathrm{~m}, 1 \mathrm{H}$, of cyclohexyl group), 5.66 (s, $1 \mathrm{H}, \mathrm{NH}$-cyclohexyl) ( $\mathrm{D}_{2} \mathrm{O}$ exchangeable), 6.80(s, $\left.1 \mathrm{H}, \mathrm{NH}-\mathrm{SO}_{2}\right)\left(\mathrm{D}_{2} \mathrm{O}\right.$ exchangeable), 7,72-8.10(m, 4H, aromatic); Anal. Calcd. for $\mathrm{C}_{21} \mathrm{H}_{17} \mathrm{Br}_{4} \mathrm{~N}_{3} \mathrm{O}_{5} \mathrm{~S}: \mathrm{C}, 33.94 ; \mathrm{H}, 2.31 ; \mathrm{N}, 5.66$; Found: C, 33.63; H, 2.72; N, 5.21.

2.1.14. N-(butylcabamoyl)-4-(4,5,6,7-tetrabromo-1,3-dioxoisoindolin-2-yl)benzenesulfonamide $\left(\mathrm{VII}_{f}\right)$ :

0.84 g, (59\%); mp: $281-282{ }^{\circ} \mathrm{C}$; IR: $2 \mathrm{NH} 3254$, CH-aliphatic 2932, 3CO $1693 \mathrm{~cm}^{-1}$; Ms: m/z $717\left(\mathrm{M}^{+}+4\right)(2.30 \%), 617\left(\mathrm{M}^{+}+4-\mathrm{CONH}-\right.$ butyl) (11.08\%), 51(100\%), Anal. Calcd. for $\mathrm{C}_{19} \mathrm{H}_{15} \mathrm{Br}_{4} \mathrm{~N}_{3} \mathrm{O}_{5} \mathrm{~S}: \mathrm{C}, 31.83 ; \mathrm{H}, 2.11 ; \mathrm{N}, 5.86$; Found: C, 31.39; H, 2.47; N, 5.54.

2.1.15.

N-(propylcabamoyl)-4-(4,5,6,7-tetrabromo-1,3-dioxoisoindolin-2-yl)benzenesulfonamide ( $\mathrm{VII}_{\mathrm{g}}$ ):

0.78 g, (56\%); mp: 294-295 ${ }^{\circ} \mathrm{C}$; IR: $2 \mathrm{NH} 3356,3264$, CH-aliphatic 2954, 3CO 1682, 1601 $\mathrm{cm}^{-1} ;{ }^{1} \mathrm{H}$ NMR (DMSO-d $\left.{ }_{6}\right): \delta 1.03\left(\mathrm{t}, 3 \mathrm{H}, \mathrm{CH}_{3}\right), 1.23\left(\mathrm{~m}, 2 \mathrm{H}, \mathrm{C}_{2} \mathrm{CH}_{3}\right), 3.45(\mathrm{t}, 2 \mathrm{H}$, $\left.\mathrm{CH}_{2} \mathrm{CH}_{2} \mathrm{CH}_{3}\right), \quad 5.60$ (s, 1H, NH-propyl) ( $\mathrm{D}_{2} \mathrm{O}$ exchangeable), 6.43(s, $\left.1 \mathrm{H}, \mathrm{NH}-\mathrm{SO}_{2}\right)\left(\mathrm{D}_{2} \mathrm{O}\right.$ exchangeable), 7.24-8.08(m, 4H, aromatic); Anal. Calcd. for $\mathrm{C}_{18} \mathrm{H}_{13} \mathrm{Br}_{4} \mathrm{~N}_{3} \mathrm{O}_{5} \mathrm{~S}: \quad \mathrm{C}, 30.75 ; \mathrm{H}$, 1.86 ; N, 5.98; Found: C, 30.31; H, 2.11; N, 6.33.

2.1.16. $\quad \mathrm{N}$-(cyclohexylcarbamoyl)-4-[(1,3-dioxoisoindolin-2-yl)methyl]benzenesulfonamide $\left(\mathrm{VII}_{h}\right)$ :

0.50 g, (57\%); mp: $183-184^{\circ} \mathrm{C}$; IR: 2NH 3328, CH-aliphatic 2929, 3CO $1626 \mathrm{~cm}^{-1} ; \mathrm{Ms}: \mathrm{m} / \mathrm{z}$ $440\left(\mathrm{M}^{+}-1\right)(5.00 \%), 235\left(\mathrm{M}^{+}\right.$- SONHCONH-cyclohexyl) (23.49\%), 56(100\%), Anal. Calcd. for $\mathrm{C}_{22} \mathrm{H}_{23} \mathrm{~N}_{3} \mathrm{O}_{5} \mathrm{~S}$ : C, 59.85; H, 5.25; N, 9.52; Found: C, 60.19; H, 5.39; N, 9.19.

2.1.17. $N$-(cyclopentylcabamoyl)-4-[(1,3-dioxoisoindolin-2-yl)methyl] benzene- sulfonamide $\left(V I I_{i}\right):$

$0.47 \mathrm{~g},(55 \%)$; mp: $180-181{ }^{\circ} \mathrm{C}$; IR: $2 \mathrm{NH} 3290$, CH-aliphatic $2952,3 \mathrm{CO} 1644,{ }^{1} \mathrm{H}$ NMR (DMSO-d $\left.\mathrm{d}_{6}\right): \delta 1.04-1.66(\mathrm{~m}, 8 \mathrm{H}$, cyclopentyl group), $3.10(\mathrm{~m}, 1 \mathrm{H}$, of cyclopentyl group), 4.70(s, 2H, $\left.\mathrm{CH}_{2}\right), 5.63\left(\mathrm{~s}, 1 \mathrm{H}, \mathrm{NH}\right.$-cyclopentyl) ( $\mathrm{D}_{2} \mathrm{O}$ exchangeable), 6.40(s, 1H, NH-SO ${ }_{2}$ ) ( $\mathrm{D}_{2} \mathrm{O}$ exchangeable), 7,46-8.14(m, 8H, aromatic); Anal. Calcd. for $\mathrm{C}_{21} \mathrm{H}_{21} \mathrm{~N}_{3} \mathrm{O}_{5} \mathrm{~S}$ : C, 59.00; H, 4.95; N, 9.83; Found: C, 59.42; H, 4.69; N, 9.52.

2.1.18. N-(butylcabamoyl)-4-[(1,3-dioxoisoindolin-2-yl)methyl]benzenesulfona- mide $\left(\mathrm{VII}_{\mathrm{j}}\right)$ :

$0.46 \mathrm{~g}$, (56\%); mp: $186-187^{\circ} \mathrm{C}$; IR: $2 \mathrm{NH} 3330$, CH-aliphatic 2950, 3CO 1644, Ms: m/z 415 $\left(\mathrm{M}^{+}\right)(2.40 \%), 316\left(\mathrm{M}^{+}\right.$- CONH-butyl) (29.33\%), 236(100\%), Anal. Calcd. for $\mathrm{C}_{20} \mathrm{H}_{21} \mathrm{~N}_{3} \mathrm{O}_{5} \mathrm{~S}$ : C, 57.82; H, 5.09; N, 10.11; Found: C, 57.48; H, 5.33; N, 10.42.

2.1.19. N-(propylcabamoyl)-4-[(1,3-dioxoisoindolin-2-yl)methyl]benzenesulfon- amide $\left(\mathrm{VII}_{k}\right)$ :

$0.47 \mathrm{~g}$, (59\%); mp: 190-191 ${ }^{\circ} \mathrm{C}$; IR: 2NH 3377,3300, 3CO 1698,1651, Ms: m/z $402\left(\mathrm{M}^{+}+1\right)$ (3.74\%), 235 ( $\mathrm{M}^{+}-\mathrm{SO}_{2} \mathrm{NHCONH}-$ propyl) $(33.24 \%), 77(100 \%)$, Anal. Calcd. for $\mathrm{C}_{19} \mathrm{H}_{19} \mathrm{~N}_{3} \mathrm{O}_{5} \mathrm{~S}: \mathrm{C}, 56.85 ; \mathrm{H}, 4.77 ; \mathrm{N}, 10.47$;Found: C, 57.08; H, 5.10; N, 10.88 . 
2.1.20. N-(cyclohexylcarbamoyl)-4-[(4,5,6,7-tetrabromo-1,3-dioxoisoindolin-2yl)methyl]benzenesulfonamide $\left(\mathrm{VII}_{l}\right)$ :

0.90 g, (60\%); mp: $305-306^{\circ} \mathrm{C}$; IR: $2 \mathrm{NH} 3348, \mathrm{CH}$-aliphatic 2937, $3 \mathrm{CO} 1695, \mathrm{Ms}: \mathrm{m} / \mathrm{z} 757$ $\left(\mathrm{M}^{+}+4\right)(3.14 \%), 115(100 \%)$, Anal. Calcd. for $\mathrm{C}_{22} \mathrm{H}_{19} \mathrm{Br}_{4} \mathrm{~N}_{3} \mathrm{O}_{5} \mathrm{~S}: \mathrm{C} \mathrm{C}, 34.90 ; \mathrm{H}, 2.53$; N, 5.55; Found: C, 35.38; H, 2.95; N, 5.78.

\subsubsection{1.}

$N$-(butylcabamoyl)-4-[(4,5,6,7-tetrabromo-1,3-dioxoisoindolin-2-yl)methyl]benzenesulfonamide (VII $\left.{ }_{m}\right)$ :

0.85 g, (59\%); mp: 303-304 ${ }^{\circ} \mathrm{C}$; IR: $2 \mathrm{NH} 3350$, CH-aliphatic 2944, 3CO $1689 \mathrm{~cm}^{-1}$; ${ }^{1} \mathrm{H}$ NMR (DMSO-d $\left.)_{6}\right): \delta 0.80\left(\mathrm{t}, 3 \mathrm{H}, \mathrm{CH}_{3}\right), 1.39\left(\mathrm{~m}, 2 \mathrm{H}, \mathrm{CH}_{2} \mathrm{CH}_{3}\right), 2.55\left(\mathrm{~m}, 2 \mathrm{H}, \mathrm{C}_{2} \mathrm{CH}_{2} \mathrm{CH}_{3}\right)$, 3.16(t, 2H, $\left.\underline{\mathrm{H}}_{2} \mathrm{CH}_{2} \mathrm{CH}_{2} \mathrm{CH}_{3}\right), 4.71\left(\mathrm{~s}, 2 \mathrm{H}, \mathrm{CH}_{2}\right.$-phthalimide), 5.55(s, 1H, NH-butyl) $\left(\mathrm{D}_{2} \mathrm{O}\right.$ exchangeable), 6.60(s, $\left.1 \mathrm{H}, \mathrm{NH}-\mathrm{SO}_{2}\right)\left(\mathrm{D}_{2} \mathrm{O}\right.$ exchangeable), 7.76-7.90(m, $4 \mathrm{H}$, aromatic); Anal. Calcd. for $\mathrm{C}_{20} \mathrm{H}_{17} \mathrm{Br}_{4} \mathrm{~N}_{3} \mathrm{O}_{5} \mathrm{~S}$ : C, 32.86; H, 2.34; N, 5.75; Found: C, 33.20; H, 2.87; N, 6.19.

2.1.22.

$\mathrm{N}$-(propylcabamoyl)-4-[(4,5,6,7-tetrabromo-1,3-dioxoisoindolin-2-yl)methyl]benzenesulfonamide $\left(\mathrm{VII}_{\boldsymbol{n}}\right)$ :

0.83g, (58\%); mp: 295-296 ${ }^{\circ} \mathrm{C}$; IR: 2NH 3373, CH-aliphatic 2933, 3CO $1665 \mathrm{~cm}^{-1} ;{ }^{1} \mathrm{H}$ NMR (DMSO-d $\mathrm{d}_{6}$ ): $\delta 0.83\left(\mathrm{t}, 3 \mathrm{H}, \mathrm{CH}_{3}\right), 1.14\left(\mathrm{~m}, 2 \mathrm{H}, \mathrm{C}_{2} \mathrm{CH}_{3}\right), 3.03\left(\mathrm{t}, 2 \mathrm{H}, \mathrm{C}_{2} \mathrm{CH}_{2} \mathrm{CH}_{3}\right), 4.50(\mathrm{~s}, 2 \mathrm{H}$, $\mathrm{CH}_{2}$-phthalimide), 5.50(s, 1H, NH-propyl) ( $\mathrm{D}_{2} \mathrm{O}$ exchangeable), $6.33\left(\mathrm{~s}, 1 \mathrm{H}, \mathrm{NH}-\mathrm{SO}_{2}\right)\left(\mathrm{D}_{2} \mathrm{O}\right.$ exchangeable), 7.53-7.89(m, $4 \mathrm{H}$, aromatic); Anal. Calcd. for $\mathrm{C}_{19} \mathrm{H}_{15} \mathrm{Br}_{4} \mathrm{~N}_{3} \mathrm{O}_{5} \mathrm{~S}: \mathrm{C}, 31.83 ; \mathrm{H}$, 2.11 ; N, 5.86;Found: C, 32.13; H, 2.43; N, 6.13.

\subsubsection{3. $N$-(cyclohexylcarbamoyl)-4-[2-(1,3-dioxoisoindolin-2-yl)ethyl]benzene- sulfonamide} $\left(V I I_{o}\right):$

$0.55 \mathrm{~g},(60 \%)$; mp: $157-159{ }^{\circ} \mathrm{C}$; IR: $2 \mathrm{NH} 3345$, CH-aliphatic $2934,3 \mathrm{CO} 1694,{ }^{1} \mathrm{H}$ NMR (DMSO-d $\left.\mathrm{d}_{6}\right): \delta 1.17-1.70\left(\mathrm{~m}, 10 \mathrm{H}\right.$, cyclohexyl group), 2.92(t, 2H, $\left.\mathrm{CH}_{2}-\mathrm{ph}\right), \quad 3.23(\mathrm{~m}, 1 \mathrm{H}$, of cyclohexyl group), 3.49(t, 2H, $\mathrm{CH}_{2}$-phthalimide), 5.54(s, $1 \mathrm{H}, \mathrm{NH}$-cyclohexyl) ( $\mathrm{D}_{2} \mathrm{O}$ exchangeable), 6.34(s, 1H, NH-SO $\left.{ }_{2}\right)$ ( $\mathrm{D}_{2} \mathrm{O}$ exchangeable), 6,99-7.83(m, 8H, aromatic); Anal. Calcd. for $\mathrm{C}_{23} \mathrm{H}_{25} \mathrm{~N}_{3} \mathrm{O}_{5} \mathrm{~S}$ : C, 60.64; H, 5.53; N, 9.22; Found: C, 60.22; H, 5.89; N, 9.65.

\subsubsection{4. $N$-(cyclopentylcabamoyl)-4-[2-(1,3-dioxoisoindolin-2-yl)ethyl]benzene- sulfonamide} $\left(\mathrm{VII}_{p}\right)$ :

$0.50 \mathrm{~g}$, (57\%); mp: $163-164{ }^{\circ} \mathrm{C}$; IR: 2NH 3333, CH-aliphatic 2940, 3CO 1694, Ms: m/z 442 $\left(\mathrm{M}^{+}+1\right)(38.08 \%), 328\left(\mathrm{M}^{+}-\mathrm{CONH}-\right.$ cyclopentyl) $(23.64 \%), 160\left(\mathrm{M}^{+}-\mathrm{CH}_{2} \mathrm{C}_{6} \mathrm{H}_{4} \mathrm{SO}_{2} \mathrm{NHCONH}-\right.$ cyclopentyl) (12\%), 29(100\%), Anal. Calcd. for $\mathrm{C}_{22} \mathrm{H}_{23} \mathrm{~N}_{3} \mathrm{O}_{5} \mathrm{~S}: \mathrm{C}, 59.85 ; \mathrm{H}, 5.25 ; \mathrm{N}, 9.52$; Found: C, 60.13; H, 5.57; N, 9.81.

2.1.25. $\quad N$-(butylcabamoyl)-4-[2-(1,3-dioxoisoindolin-2-yl)ethyl]benzenesulfona- mide $(\text { VII })_{\text {): }}$

$0.47 \mathrm{~g}$, (55\%); mp: $186-187^{\circ} \mathrm{C}$; IR: $2 \mathrm{NH} 3422,3322, \mathrm{CH}$-aliphatic $2945,3 \mathrm{CO} 1680, \mathrm{Ms}: \mathrm{m} / \mathrm{z}$ $429\left(\mathrm{M}^{+}\right)(2.49 \%), 160\left(\mathrm{M}^{+}-\mathrm{CH}_{2} \mathrm{C}_{6} \mathrm{H}_{4} \mathrm{SO}_{2} \mathrm{NHCONH}-\right.$ butyl) (10.11\%), 77(100\%), Anal. Calcd. for $\mathrm{C}_{21} \mathrm{H}_{23} \mathrm{~N}_{3} \mathrm{O}_{5} \mathrm{~S}$ : C, 58.73; H, 5.40; N, 9.78; Found: C, 59.11; H, 5.89; N, 10.11.

2.1.26. $\quad \mathrm{N}$-(propylcabamoyl)-4-[2-(1,3-dioxoisoindolin-2-yl)ethyl]benzenesulfo- namide $\left(\mathrm{VII}_{r}\right)$ :

0.46 g, (55\%); mp: $213-214{ }^{\circ} \mathrm{C}$; IR: $2 \mathrm{NH} 3329$, CH-aliphatic 2925, 3CO $1616 \mathrm{~cm}^{-1}$; ${ }^{1} \mathrm{H}$ NMR (DMSO-d $\left.\mathrm{d}_{6}\right): \delta 0.80\left(\mathrm{t}, 3 \mathrm{H}, \mathrm{CH}_{3}\right), 1.10\left(\mathrm{~m}, 2 \mathrm{H}, \mathrm{CH}_{2} \mathrm{CH}_{3}\right), 2.90\left(\mathrm{t}, 2 \mathrm{H}, \mathrm{CH}_{2}-\mathrm{ph}\right), \quad 3.01(\mathrm{t}$, $\left.2 \mathrm{H}, \quad \mathrm{CH}_{2} \mathrm{CH}_{2} \mathrm{CH}_{3}\right), \quad 3.87\left(\mathrm{t}, 2 \mathrm{H}, \mathrm{CH}_{2}\right.$-phthalimide), 5.55(s, 1H, NH-propyl) ( $\mathrm{D}_{2} \mathrm{O}$ exchangeable), 6.23(s, 1H, NH-SO $\left.{ }_{2}\right)\left(\mathrm{D}_{2} \mathrm{O}\right.$ exchangeable), 7.80-8.33(m, 8H, aromatic); Anal. Calcd. for $\mathrm{C}_{20} \mathrm{H}_{21} \mathrm{~N}_{3} \mathrm{O}_{5} \mathrm{~S}$ : C, 57.82; H, 5.09; N, 10.11; Found: C, 57.53; H, 5.33; N, 10.46. 
2.1.27. N-(cyclohexylcarbamoyl)-4-[2-(4,5,6,7-tetrabromo-1,3-dioxoisoindolin-2yl)ethyl]benzenesulfonamide (VII $)$ :

0.88 g, (57\%); mp: $246-247{ }^{\circ} \mathrm{C}$; IR: $2 \mathrm{NH} 3324, \mathrm{CH}$-aliphatic 2928, 3CO 1656, ${ }^{1} \mathrm{H}$ NMR (DMSO-d $\left.{ }_{6}\right): \delta 1.02-1.61\left(\mathrm{~m}, 10 \mathrm{H}\right.$, cyclohexyl group), 2.90(t, $\left.2 \mathrm{H}_{2} \mathrm{CH}_{2}-\mathrm{ph}\right), 3.16(\mathrm{~m}, 1 \mathrm{H}$, of cyclohexyl group), 3.66(t, 2H, $\mathrm{CH}_{2}$-phthalimide), 5.60(s, $1 \mathrm{H}, \mathrm{NH}$-cyclohexyl) ( $\mathrm{D}_{2} \mathrm{O}$ exchangeable), 6.44(s, 1H, NH-SO $\mathrm{S}_{2}$ ( $\mathrm{D}_{2} \mathrm{O}$ exchangeable), 7,44-7.76(m, 4H, aromatic); Anal. Calcd. for $\mathrm{C}_{23} \mathrm{H}_{21} \mathrm{Br}_{4} \mathrm{~N}_{3} \mathrm{O}_{5} \mathrm{~S}$ : C, 35.82; H, 2.74; N, 5.45; Found: C, 35.65; H, 2.73; N, 5.87 .

2.1.28. $\mathrm{N}$-(butylcabamoyl)-4-[2-(4,5,6,7-tetrabromo-1,3-dioxoisoindolin-2-yl) ethyl]benzenesulfonamide $\left(\mathrm{VII}_{t}\right)$ :

$0.53 \mathrm{~g},(56 \%)$; mp: $219-220{ }^{\circ} \mathrm{C}$; IR: $2 \mathrm{NH} 3342, \mathrm{CH}$-aliphatic $2943,3 \mathrm{CO} 1665 \mathrm{~cm}^{-1}$; ${ }^{1} \mathrm{H}$ NMR (DMSO-d $\left.\mathrm{d}_{6}\right): \delta 0.83\left(\mathrm{t}, 3 \mathrm{H}, \mathrm{CH}_{3}\right), 1.23\left(\mathrm{~m}, 2 \mathrm{H}, \mathrm{CH}_{2} \mathrm{CH}_{3}\right), 1.90\left(\mathrm{~m}, 2 \mathrm{H}, \mathrm{C}_{2} \mathrm{CH}_{2} \mathrm{CH}_{3}\right)$, 2.90(t, 2H, $\mathrm{CH}_{2}$-ph), 3.20(t, $\left.2 \mathrm{H}, \mathrm{CH}_{2} \mathrm{CH}_{2} \mathrm{CH}_{2} \mathrm{CH}_{3}\right), 3.45\left(\mathrm{t}, 2 \mathrm{H}, \mathrm{CH}_{2}\right.$-phthalimide), 5.51(s, $1 \mathrm{H}, \mathrm{NH}$-butyl) ( $\mathrm{D}_{2} \mathrm{O}$ exchangeable), 6.45(s, 1H, NH-SO $)_{2}\left(\mathrm{D}_{2} \mathrm{O}\right.$ exchangeable), 7.287.77(m, 4H, aromatic); Anal. Calcd. for $\mathrm{C}_{21} \mathrm{H}_{19} \mathrm{Br}_{4} \mathrm{~N}_{3} \mathrm{O}_{5} \mathrm{~S}: \mathrm{C}, 33.85 ; \mathrm{H}, 2.57 ; \mathrm{N}, 5.64$; Found: C, 33.50; H, 2.92; N, 6.01.

2.1.29. N-(propylcabamoyl)-4-[2-(4,5,6,7-tetrabromo-1,3-dioxoisoindolin-2-yl) ethyl]benzenesulfonamide $\left(\mathrm{II}_{u}\right)$ :

$0.81 \mathrm{~g}$, (54\%); mp: $211-212{ }^{\circ} \mathrm{C}$; IR: $2 \mathrm{NH} 3310$, CH-aliphatic 2920, 3CO $1615 \mathrm{~cm}^{-1}$; ${ }^{1} \mathrm{H}$ NMR (DMSO-d $\left.\mathrm{d}_{6}\right): \delta$ 0.98(t, 3H, $\left.\mathrm{CH}_{3}\right), 1.60\left(\mathrm{~m}, 2 \mathrm{H}, \mathrm{CH}_{2} \mathrm{CH}_{3}\right), 2.88\left(\mathrm{t}, 2 \mathrm{H}, \mathrm{CH}_{2}-\mathrm{ph}\right), \quad 3.17(\mathrm{t}$, $\left.2 \mathrm{H}, \quad \mathrm{CH}_{2} \mathrm{CH}_{2} \mathrm{CH}_{3}\right), \quad 3.66\left(\mathrm{t}, 2 \mathrm{H}, \mathrm{CH}_{2}\right.$-phthalimide), 5.66(s, $1 \mathrm{H}, \mathrm{NH}$-propyl) $\left(\mathrm{D}_{2} \mathrm{O}\right.$ exchangeable), 6.51(s, $\left.1 \mathrm{H}, \mathrm{NH}-\mathrm{SO}_{2}\right)\left(\mathrm{D}_{2} \mathrm{O}\right.$ exchangeable), 7.44-7.77(m, $4 \mathrm{H}$, aromatic); Anal. Calcd. for $\mathrm{C}_{20} \mathrm{H}_{17} \mathrm{Br}_{4} \mathrm{~N}_{3} \mathrm{O}_{5} \mathrm{~S}$ : C, 32.86; H, 2.34; N, 5.75; Found: C, 33.10; H, 2.73; N, 6.10 .

\section{PHARMACOLOGY}

\subsection{Principle of Antihyperglycemic test}

The test based on the method used by Ramsey et al. (Ramsey, et al., 2007) where the compounds to be tested or the standard (gliclazide) were given by oral route to groups of hyperglycemic adult male rats. After administration by three hours, the blood glucose level was determined and compared with standard.

\subsection{Materials and Methods}

Adult male rats $(72 \pm 10$ days of age, weight ranging between 100 to $150 \mathrm{~g})$ were injected intraperitoneally with streptozotocin (STZ), (60 mg/kg, Sigma) dissolved in $0.9 \%$ Sodium citrate buffer ( $\mathrm{pH} 4.5$ ) (Tanko, et al., 2008).

Blood glucose levels were measured 48-72 hrs after STZ administration and a value $>350$ $\mathrm{mg} / \mathrm{dl}$ was considered to be diabetic. The diabetic rats were divided into three groups (i) drug treated group comprised of 12 subgroups for 12 test compounds, (ii) standard treated group and (iii) vehicle treated group. Each group as well as sub-group was comprised of 6 animals. The tested compounds as well as the standard drug were suspended in 5\% gum acacia and administered by oral route at a dose of $200 \mathrm{mg} / \mathrm{kg}$. After three hours, blood was drawn from the tail of conscious rats and the glucose content was estimated with using the (ACCU-CHEK Active) instrument and results were reported as $\mathrm{mg} / \mathrm{dl}$ ). 
Table 1: Antihyperglycemic activity of some selected newly synthesized compounds and reference drug

\begin{tabular}{|c|c|c|}
\hline Comp. No. & $\begin{array}{c}\text { Serum glucose } \\
(\mathrm{mg} / \mathrm{dL})\end{array}$ & $\begin{array}{c}\text { Serum glucose } \\
\text { reduction }(\%)\end{array}$ \\
\hline $\mathrm{VII}_{\mathrm{a}}$ & 259 & $40 \%$ \\
\hline $\mathrm{VII}_{\mathrm{d}}$ & 267 & $38 \%$ \\
\hline $\mathrm{VII}_{\mathrm{e}}$ & 302 & $30 \%$ \\
\hline $\mathrm{VII}_{\mathrm{h}}$ & 146 & $44 \%$ \\
\hline $\mathrm{VII}_{\mathrm{i}}$ & 250 & $42 \%$ \\
\hline $\mathrm{VII}_{\mathrm{l}}$ & 293 & $32 \%$ \\
\hline $\mathrm{VII}_{\mathrm{o}}$ & 207 & $52 \%$ \\
\hline $\mathrm{VII}_{\mathrm{p}}$ & 224 & $48 \%$ \\
\hline $\mathrm{VII}_{\mathrm{q}}$ & 237 & $45 \%$ \\
\hline $\mathrm{VII}_{\mathrm{r}}$ & 161 & $44 \%$ \\
\hline $\mathrm{VII}_{\mathrm{s}}$ & 293 & $32 \%$ \\
\hline $\mathrm{VII}_{\mathrm{t}}$ & 289 & $33 \%$ \\
\hline Diabetic control & 432 & - \\
\hline Refrence $($ Gliclazide $)$ & 211 & $51 \%$ \\
\hline
\end{tabular}

\section{STRUCTURE-ACTIVITY CORRELATION}

Compounds belong to the unsubstituted isoindoline-1,3-dione proved to be more active than tetrabromo substituted one. Insertion of a one carbon spacer between the Nterminus of the isoindoline-1,3-dione and the phenyl moiety increased the hypoglycemic activity. Extending the spacer length to two carbons distance (as $\mathbf{V I I}_{\mathbf{0}}, \mathbf{V I I}_{\mathbf{p}}, \mathbf{V I I}_{\mathbf{q}}$ and $\mathbf{V I I} \mathbf{r}$ ) produced the most active agent in this work, with $52 \%, 48 \%$ and $45 \%$ reduction, respectively. It seemed that two carbons distance favor the interaction with the enzymatic receptors involved. The bulky and long chain alkyl groups on the N-substitution of the sulfonamide moiety produced higher activity than the less bulky and short alkyl one(cyclohexyl and butyl groups are more active than cyclopentyl and propyl groups respectively).

\section{CONCLUSION}

In the present study, certain new cyclic-imides isoindoline-1,3-diones and their isosters tetrabromoisoindole-1,3- diones bearing aryl sulfonyl urea moieties were synthesized. All of the synthesized Compounds N-(cyclohexylcarbamoyl) -4-[2-(1,3dioxoisoindolin-2-yl)ethyl]benzenesulfonamide (VII $), N$-(cyclopentyl cabamoyl) -4- [2-(1,3dioxoisoindolin- 2- yl) ethyl] benzenesulfonamide (VII $) \quad N$-(butylcabamoyl)-4-[2-(1,3dioxoisoindolin-2-yl)ethyl] benzenesulfonamide (VII $\mathbf{q}_{\mathbf{q}}, N$-(cyclohexylcarbamoyl)-4-[(1,3dioxoisoindolin-2-yl)methyl] benzene sulfonamide ( $\left.\mathbf{V I I}_{\mathbf{h}}\right)$ and $N$-(propylcabamoyl)-4-[2-(1,3dioxoisoindolin-2-yl)ethyl] benzenesulfonamide (VII $)_{\mathbf{r}}$ showed 52, 48, 45, 44 and 44\% reduction in serum glucose level, respectively. Gliclazide showed only $51 \%$ reduction.

\section{ACKNOWLEDGMENT}

The author would like to thank the Pharmacology Department, Faculty of Pharmacy, Al-Azhar University for performing antihyperglycemic testing. 


\section{REFERENCES:}

Abdel-Aziz A.M., El-Azab A., Attia S.M., Al-Obaid A.M., Al-Omar M.A. and El-Subbagh H.I., (2011): European Journal of Medicinal Chemistry; 46:4324-9.

Chan S.H., Lam K.H. , Chui C.H., Gambari R., Yuen M.C. and Wong R.S., (2009): European Journal of Medicinal Chemistry; 44:2736-2740.

Fertig B.J., Simmon S.A. and Marten M.B., (1995): Diabetes; 95:1468-9.

Hashimoto, Y., Curr. Med. Chem. 1998; 5:163.

http://www.afro.who.int/en/clusters-a-programmes/dpc/non-communicable-diseasesmanagementndm/programme-components/diabetes-mellitus.html, Accessed 6/2013.

http://diabetesmanager.pbworks.com/w/page/17680289/Oral\%20 Pharma cological\% 20Agents\% 20for\% 20Type\% 202\% 20Diabetes, Accessed 9/ 2013.

Ibrahim M.K., Ayyad R.R., Aladl K. and Rashed M., Al-Azhar j. of pharm. Science 2010; 42:305-322.

$\operatorname{Kar} A$, (2006): Medicinal chemistry, $4^{\text {th }}$ edition, 2006;678.

Kim Y., Yang H., Park K., Koh D. and Lim Y., (2002): Agric. Chem. Biotechnol.; 45(3): $141-6$

Korytkowski M.T., (2004): Pharmacotherapy; 24:606-620.

Mahapatra S.P., Ghode P., Jain D.K., Chaturvedi S.C., Maiti B.C. and Maity T.K., (2010): j. pharm. Sci., \& Res.; 2(9): 567-8.

Miyachi H., Ogasawara A., Azuma A. and Hashimoto Y., (1007): Bioorganic \& Medicinal Chemistry; 5:2095-2102.

Nadendla R.R., (2005): Principle of organic medicinal chemistry, $1^{\text {st }}$ edition; 274-278.

Pascale R., Carocci A., Catalano A., Lentini G., Spagnoletta A., Cavalluzzi M. M., Santis F.D., Palma A.D., Scalera V. and Franchini C., (2010): Bioorganic \& Medicinal Chemistry;18: 5903-5914

Ramsey D.J., Ripps H. and Qianab H., (2007): Exp Eye Res.; 85: 413 -422.

Santos J.L., Yamasaki P.R., Chin C.M., Takashi C.H., Pavan F.R. and Leite C.F., (2009): Bioorganic \& Medicinal Chemistry; 17: 3795-9.

Tanko Y., Yerim M., Mahdi M.A., Yaro A.H., Musa K.Y. and Mohammed A., (2008): International Journal of Applied Research in Natural Products; 1:32-6.

Yang Y.J., Yang Y.N., Jiang J.S., Feng Z.M., Liu H.Y. and Pan X.D., (2010): Chinese Chemical Letters; 21:902- 4.

Zhao Q., Yang G., Mei X., Yuan H. and Ning J., (2009): Pesticide Biochemistry and Physiology; 95:131- 4. 
تشييد بعض المشتقات الجديدة من نواة الأيزوإندولين ا, بـ دايون لإختبار ها كمضادات لزيادة السكر

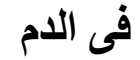

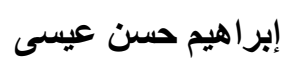

قسم الكيمياء الصيدلية كلية الصبدلة (بنين) جامعة الأزهر القاهرة

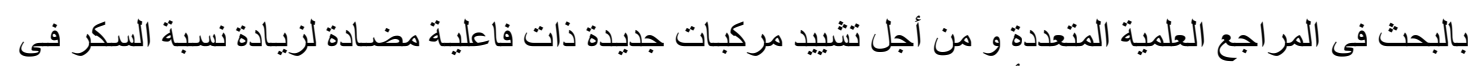

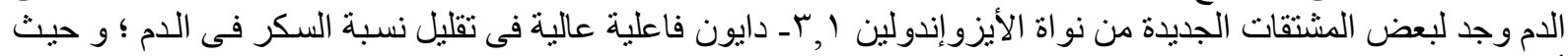

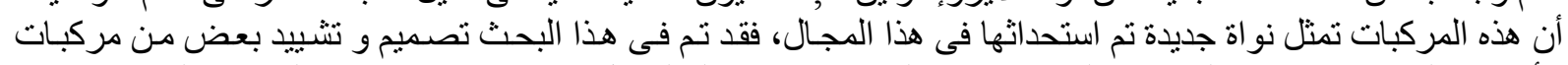

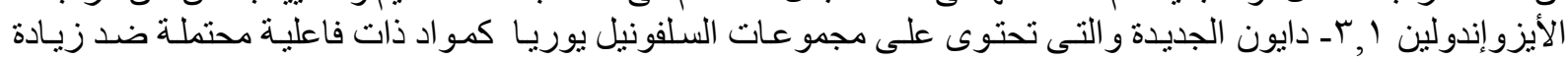

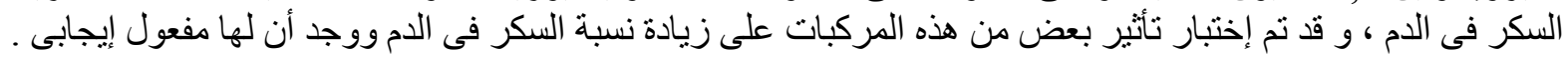

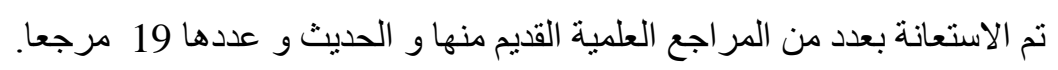

